\title{
CASO AlbAm S.A.
}

\author{
Ricardo Goulart Serra \\ ricardo.serra@usp.br \\ Insper Ibmec/SP e Fecap - São Paulo, SP / Brasil
}

\begin{abstract}
http://dx.doi.org/10.1590/1413-2311.0812014.54869
Recebido em 26/12/2014

Aprovado em 21/12/2015

Disponibilizado em 31/12/2015

Avaliado pelo sistema "double blind review"

Revista Eletrônica de Administração

Editor: Luís Felipe Nascimento

ISSN 1413-2311 (versão "on line")

Editada pela Escola de Administração da Universidade Federal do Rio Grande do Sul.

Periodicidade: Quadrimestral

Sistema requerido: Adobe Acrobat Reader
\end{abstract}

\section{RESUMO}

Os analistas financeiros da AlbAm, uma empresa do setor de autopeças, devem avaliar duas opções de aquisições (Cardblue e Garciadores). O objetivo do caso é servir de oportunidade para os alunos vivenciarem uma decisão de investimentos por meio do lucro econômico. Uma análise superficial certamente levará a decisão equivocada. Levando-se todos os aspectos do caso em consideração, chegar-se-á a uma solução que pode surpreender alguns alunos, uma vez que a empresa melhor gerida não será a melhor oportunidade de investimento. Também se pode aproveitar o caso para discutir a superficialidade da adoção do EBITDA como métrica de gestão comparativamente a uma métrica de valor agregado (lucro econômico) - a melhor solução também não é aquela que gera o maior aumento em EBITDA. O caso pode ser usado em disciplinas de finanças (finanças corporativas ou avaliação de empresas) em cursos de graduação, pós-graduação ou especialização. Todas as empresas e pessoas relatadas neste caso são fictícias.

Palavras-Chave: lucro econômico; lucro residual; EVA ${ }^{\circledR}$; avaliação de empresas; caso de ensino

\section{CASE AlbAm S.A.}

\begin{abstract}
The financial analysts of AlbAm, a company in the auto parts industry, have to evaluate two acquisition opportunities (Cardblue and Garciadores). The objective of the case is to serve as an opportunity for students to experience an investment decision through economic profit. A superficial analysis will certainly lead to a mistaken decision. Considering all aspects of the case, one will come up with a solution that may surprise some students as the best managed company will not be the best investment opportunity. The case can also be used to discuss the superficiality of the adoption of EBITDA as a managerial metric compared to a value added metric (economic profit) - the best solution is not the one that generates the largest increase in REAd | Porto Alegre - Edição 82 - № 3 - setembro/dezembro 2015 - p. 782-795
\end{abstract}




\section{Ricardo Goulart Serra}

EBITDA. The case can be used in finance courses (corporate finance or valuation) in undergraduate, graduate or specialization programs. All companies and people described herein are fictitious.

Keywords: economic profit; residual income; EVA ${ }^{\circledR}$; valuation; teaching case

\section{CASO AlbAm S.A.}

\section{RESUMEN}

Los analistas financieros de Albam, una empresa en el sector de autopartes, deben evaluar dos oportunidades de adquisición (Cardblue y Garciadores). El objetivo del caso es la de servir como una oportunidad para que los estudiantes experimentan una decisión de inversión a través de lucro económico. Un análisis superficial sin duda dará lugar a una decisión equivocada. Teniendo en cuenta todos los aspectos del caso, se llegará a una solución que puede sorprender a algunos estudiantes como la empresa mejor administrada no será la mejor oportunidad de inversión. También puede tomar el caso para discutir la superficialidad de la adopción de EBITDA como una métrica de gestión en comparación con una métrica de valor agregado (lucro económico) - la mejor solución no es la que genera el mayor incremento en el EBITDA. El caso se puede utilizar en las disciplinas de finanzas (finanzas corporativas o de valoración de empresas) en pregrado, postgrado o especialización. Todas las empresas y las personas reportadas son ficticios.

Palabras clave: lucro económico; lucro residual; EVA; valoración de empresa; caso de enseñanza

\section{INTRODUÇÃO}

Em 31 de março de 2014, segunda-feira, Luiza Lion, Diretora de Fusões e Aquisições da AlbAm, filial brasileira de uma importante empresa do setor de autopeças, passa para seu mais talentoso funcionário, Alberto Gaderra, a tarefa de montar uma apresentação com as devidas recomendações sobre a potencial compra de 2 empresas do setor.

"Alberto, temos que analisar a potencial compra da Carblue S.A. ou da Garciadores S.A.. Preciso que você prepare uma apresentação para a reunião de conselho que acontecerá na sexta-feira. Peço que enfoque o lucro econômico, tendo em vista que este é o indicador que o conselho de administração, que tem como missão defender os interesses dos acionistas, tem enfatizado ultimamente", disse Luiza.

"Pode deixar, Luiza. Acho que depois que convencemos o conselho de que olhar para EBITDAé pobre comparativamente a olhar para lucro econômico, realmente, não houve uma análise de investimentos em que eles não perguntassem os impactos no mesmo. Acho que eles se convenceram da importância de olhar o lucro econômico quando recomendamos não fazer

REAd | Porto Alegre - Edição 82 - N 3 - setembro/dezembro 2015 - p. 782-795 


\section{CASO AlbAm S.A.}

o projeto Altaira, que realmente aumentaria o EBITDA da AlbAm, mas a necessidade de capital era muito alta para o benefício gerado, o que impactaria negativamente no valor da empresa", respondeu Alberto.

"Como a matriz está com baixo apetite para investir no Brasil, temos que olhar se faz sentido alguma compra e qual das duas seria melhor. O Marco Sena já está pesquisando as informações e está instruído a ajudá-lo. Qualquer dúvida sobre a parte operacional, fale com a Bruna Hill", ponderou Luiza, já de saída.

\section{Reunindo as informações sobre a Carblue e a Garciadores e começando os cálculos}

No dia seguinte, Alberto e Marco reuniram-se com as demonstrações financeiras de 31 de dezembro de 2013 das empresas em mãos. As informações da Carblue foram obtidas no mercado, uma vez que a empresa é listada, e as informações da Garciadores, que não é listada, foram fornecidas pela própria empresa após a AlbAm ter assinado um acordo de confidencialidade. Marco já havia calculado alguns índices financeiros.

"A primeira tarefa é calcular o lucro econômico de cada uma das empresas, inclusive da AlbAm, para 2013", disse Marco.

"Concordo com você. Repare que a Garciadores, segundo a Bruna, nossa diretora de operações, usa uma tecnologia diferente da nossa, o que torna a sua operação mais eficiente, mas, também, mais arriscada. Note que o índice 'dias de estoque' da empresa é melhor do que o nosso e o da Carblue. A Luiza pediu para considerarmos um custo de capital 3 pontos percentuais maior do que o nosso, por causa deste risco maior. Repare que a Carblue, por outro lado, tem margens menores do que as nossas. Sempre ouvimos o diretor de Relações com Investidores da empresa minimizar este problema falando que eles têm um custo de capital mais baixo do que o seus peers (inclusive o nosso) em 2 pontos percentuais. Vamos trabalhar com esta informação para o cálculo do lucro econômico individual e depois pensamos em como encaminhar os demais pontos na hora de analisarmos as empresas combinadas. Particularmente, não acredito neste custo de capital da Carblue", disse Alberto.

Marco perguntou se o custo de capital usado nas análises da AlbAm continuava 19,5\% e a alíquota de imposto continuava $34 \%$, ao que o Alberto respondeu afirmativamente.

\section{Analisando os primeiros cálculos}

REAd | Porto Alegre - Edição 82 - № 3 - setembro/dezembro 2015 - p. 782-795 


\section{Ricardo Goulart Serra}

Com base no lucro econômico das empresas individuais, Marco dirigiu-se a Alberto comentando:

"Repare que a Carblue destruiu valor em 2013 enquanto a Garciadores gerou valor. Acho que desta vez vamos sugerir a compra da Garciadores e a Bruna finalmente terá acesso a esta tão falada tecnologia...".

"Calma, meu jovem padawan", interrompeu-o Alberto, fãs que eram de Star Wars. "Vamos calcular o lucro econômico das duas possíveis combinações: AlbAm + Carblue e AlbAm + Garciadores. Para tanto, considere para a Carblue que a empresa combinada terá a nossa margem bruta e o nosso custo de capital. Para as contas de balanço, vendas líquidas e despesas operacionais considere que a empresa combinada será a soma 'simples' das duas empresas. Com relação a Garciadores, considere que a empresa combinada terá a margem bruta e o custo de capital da Garciadores. Para as contas de balanço, vendas líquidas e despesas operacionais, também trabalhe com a soma 'simples' das duas empresas, exceto para a conta de estoque que, segundo conversa que tive com a Bruna, deve ser calculada com o índice 'dias de estoque' da Garciadores aplicado ao CPV da empresa combinada", instruiu Alberto.

\section{Revisitando a análise e apresentando os rascunhos para a Luiza}

Reunidos pela terceira vez em dois dias, Alberto e Marco analisavam os números.

"Acho que agora temos elementos suficientes para tirarmos conclusões preliminares. Vamos mostrar o que já temos para a Luiza, para que ela nos dê as últimas diretrizes para montarmos a apresentação", sugeriu Alberto.

Adentraram a sala da Luiza e entregaram-lhe o material. Após uma boa olhada, Luiza comentou:

"Meninos, temos uma boa parte da análise bem encaminhada. Parabéns! Vejam que os resultados preliminares nos ajudam a combater a ideia da presidência de que a tecnologia da Garciadores é boa. Eu gostaria de ter, em adição ao que vocês já produziram, a análise da AlbAm + Garciadores considerando, para a empresa combinada, a nossa margem bruta, o nosso custo de capital e o nosso índice 'dias de estoque'."

"Ótima ideia, consideremos também o cenário em que a tecnologia 'sobrevivente' será a nossa e não a deles!", exclamou Marco.

"Isto mesmo. Feito isto, calculem o preço das empresas considerando que o lucro

econômico será constante para sempre, como temos feito ultimamente, montem a REAd | Porto Alegre - Edição 82 - N 3 - setembro/dezembro 2015 - p. 782-795 


\section{CASO AlbAm S.A.}

apresentação e façam a recomendação final. Qualquer outra necessidade, avisem-me. Caso contrário, nos reunimos aqui na quinta-feira à tarde para revisarmos o material para a reunião de sexta-feira", finalizou Luiza.

No caminho de volta para suas mesas, Alberto comentou:

"Parece que deixaremos a Bruna feliz, pois pode ser que consigamos mostrar que a tecnologia que adotamos é melhor - do ponto de vista financeiro - do que a tecnologia da Garciadores, tão elogiada pelo nosso presidente Nicolau Bridges."

"É verdade. O que ela quis dizer com considerar lucro econômico constante para sempre?", respondeu Marco, emendando uma nova pergunta.

"Vamos calcular o valor das empresas (individuais e combinadas) por meio da soma do capital investido com o valor presente da agregação de valor, sendo que a agregação de valor será calculada pelo valor presente dos lucros econômicos futuros. Nesta parte é que consideraremos o lucro econômico constante para sempre, sem crescimento. Como nossas premissas já são reais e não nominais, então ficará fácil calcular o valor de cada empresa", explicou Alberto.

"Esta parte é novidade para mim", comentou Marco, ansioso para colocar estes novos conhecimentos em prática, fazer as últimas análises e finalizar o trabalho com uma recomendação.

REAd | Porto Alegre - Edição 82 - N 3 - setembro/dezembro 2015 - p. 782-795 


\section{Ricardo Goulart Serra}

ANEXO 1 - Tabelas

Tabela 1 - Principais informações do balanço das empresas AlbAm, Carblue e Garciadores em 31 de dezembro de 2013.

\begin{tabular}{lccc} 
[R Milhões] & AlbAm & Carblue & Garciadores \\
\cline { 2 - 4 } Contas do Balanço & & & \\
Caixa Operacional & 40,0 & 7,5 & 11,0 \\
Contas a Receber & 65,0 & 16,0 & 20,0 \\
Estoque & 45,0 & 11,5 & 12,5 \\
Imobilizado & 170,0 & 43,0 & 53,0 \\
& & & \\
Contas a Pagar & 35,0 & 9,0 & 10,7 \\
Impostos a Pagar & 10,0 & 2,0 & 3,0 \\
Salários e Enc. a Pagar & 5,0 & 2,0 & 2,3
\end{tabular}

Fonte: Elaborada pelo autor.

Tabela 2 - Principais informações das Demonstrações de Resultados das empresas AlbAm, Carblue e Garciadores em 31 de dezembro de 2013. A depreciação no período foi de $\mathrm{R} \$ 8,5 \mathrm{MM}, \mathrm{R} \$ 2,2 \mathrm{MM}$ e R\$ 2,7MM para a AlbAm, Carblue e Garciadores. Elaborada pelo autor.

[R \$ Milhões]

\section{DRE Parcial}

Vendas Líquidas

(-) CPV

(=) Resultado Bruto

(-) Despesas Operacionais

(=) Resultado Operacional
AlbAm

Carblue

Garciadores

$\begin{array}{ccc}800,0 & 200,0 & 250,0 \\ 600,0 & 154,0 & 185,0 \\ 200,0 & 46,0 & 65,0 \\ 115,0 & 29,0 & 36,0 \\ 85,0 & 17,0 & 29,0\end{array}$

Fonte: Elaborada pelo autor

Tabela 3 - Índices de análise financeira selecionados (índices 'dias de recebível', 'dias de estoque' e 'dias de fornecedores' calculados com 365 dias). Elaborada pelo autor.

Dias de Recebível

Dias de Estoque

Dias de Fornecedores

Giro de Ativo Fixo

Margem Bruta

Margem Operacional

\begin{tabular}{ccc} 
AlbAm & Carblue & Garciadores \\
\hline 29,7 & 29,2 & 29,2 \\
27,4 & 27,3 & 24,7 \\
21,3 & 21,3 & 21,1 \\
4,7 & 4,7 & 4,7
\end{tabular}

$\begin{array}{ccc}25,0 \% & 23,0 \% & 26,0 \% \\ 10,6 \% & 8,5 \% & 11,6 \%\end{array}$

Fonte: Elaborada pelo autor 


\section{CASO AlbAm S.A.}

\section{ANEXO 2 - Preparação para o Caso AlbAm S.A.}

Faça uma leitura prévia do caso e responda as questões abaixo:

1. Qual a missão do Alberto e do Marco?

2. Calcule o lucro econômico da AlbAm, da Carblue e da Garciadores.

3. Recalcule os índices apresentados na tabela 3.

4. Quais informações adicionais você pensa precisar para poder fazer a recomendação para o conselho de administração? Explique detalhadamente como esta informação adicional será usada na sua análise, ou seja, para quê você precisa dela (não devemos incomodar nossos superiores, parceiros ou clientes com demandas desnecessárias).

\section{Orientação Geral}

O caso apoia-se em duas premissas simplificadoras, as quais têm por objetivo simplificar a execução e favorecer as discussões:

1. Cada empresa será analisada com base em apenas 1 ano histórico - idealmente seriam necessários mais anos para esta análise (de 3 a 5 anos).

2. Cada empresa será projetada considerando que as suas características não mudarão ao longo dos anos (ativos, margens, retornos etc.) exceto por uma mudança pontual inicial que depende da tecnologia a ser adotada, conforme relata o caso. Desta forma, as empresas são consideradas perpetuidades desde o ano 1 de projeção. Idealmente as projeções considerariam alterações nestes parâmetros ao longo de um horizonte de projeções (de 5 a 10 anos) e em seguida considerar-se-ia uma perpetuidade (com ou sem crescimento). 
Ricardo Goulart Serra

\section{ANEXO 3 - Notas de Ensino (idealmente, não disponibilizar para os alunos/participantes)}

\section{Objetivo do Caso}

Este é um caso para a prática do conceito de lucro econômico para ser aplicado em cursos de graduação, pós-graduação ou especialização. O caso leva os alunos, na posição de analistas financeiros, a (i) calcular o lucro econômico de diversas empresas e suas possíveis combinações, (ii) avaliar empresas a partir do lucro econômico e (iii) decidir pela melhor opção de investimento. Pode ser que alguns alunos/participantes fiquem surpresos com a recomendação final, pois, neste caso, notarão que a empresa melhor gerida não é a melhor opção de investimento.

\section{Pré-requisitos}

Os alunos/participantes já devem conhecer a teoria para o cálculo do lucro econômico (Ehrhardt e Brigham, 2012, p. 67 e Berk e DeMarzo, 2008, p. 189). Também pode ser exigido que os alunos/participantes saibam calcular o valor de uma empresa a partir do lucro econômico - para a resolução integral do caso, este cálculo é necessário. No entanto, isto pode ser visto na própria aula da aplicação do caso.

\section{Preparação para o caso}

Deve ser solicitado aos alunos/participantes que leiam o caso anteriormente à aula de sua aplicação. Pode ser pedido aos mesmos que respondam o questionário do Anexo 2, ao qual o professor/instrutor pode adicionar ou eliminar perguntas.

\section{Plano de aula sugerido}

Para a aplicação do caso após a leitura e o trabalho prévio indicado acima, sugere-se a seguinte divisão de tempo para a sua aplicação (1h40 de duração):

REAd | Porto Alegre - Edição 82 - № 3 - setembro/dezembro 2015 - p. 782-795 
CASO AlbAm S.A.

\begin{tabular}{|l|c|}
\hline Entendimento do caso & $5 \mathrm{~min}$ \\
\hline Cálculo do lucro econômico das empresas individuais (correção) & $15 \mathrm{~min}$ \\
\hline Discussão sobre EBITDA ou lucro econômico & $15 \mathrm{~min}$ \\
\hline Melhor investimento seria comprar a empresa melhor gerida? & $10 \mathrm{~min}$ \\
\hline $\begin{array}{l}\text { Cálculo do lucro econômico das empresas combinadas (cenário } \\
\text { tecnologia 'sobrevivente' ser Garciadores) }\end{array}$ & $15 \mathrm{~min}$ \\
\hline Melhor investimento seria comprar a empresa melhor gerida? & $10 \mathrm{~min}$ \\
\hline $\begin{array}{l}\text { Cálculo do lucro econômico AlbAm + Garciadores (cenário tecnologia } \\
\text { 'sobrevivente' ser AlbAm) }\end{array}$ & $10 \mathrm{~min}$ \\
\hline Melhor investimento seria comprar a empresa melhor gerida? & $10 \mathrm{~min}$ \\
\hline Conclusões & $10 \mathrm{~min}$ \\
\hline
\end{tabular}

\section{Questões para discussões}

EBITDA versus Lucro Econômico:

Pode-se aproveitar o diálogo da Introdução e discutir se EBITDA é melhor ou pior do que lucro econômico como indicador, por exemplo, de avaliação de performance dos gestores (Copeland et al, 2005, p. 471).

Qual empresa gera mais valor / é melhor gerida:

A partir do cálculo do lucro econômico das empresas individuais, pode-se discutir: Qual delas é melhor/pior gerida? Qual delas agrega mais/menos valor?

Obs.: Pode ser interessante enfatizar que o maior lucro econômico indica qual agrega mais valor (maior lucro econômico) e não qual é melhor gerida (maior ROIC).

\section{Qual empresa é a melhor candidata - parte 1:}

Olhando para os lucros econômicos individuais, discutir: Qual aparentemente é a melhor candidata? A indicação de qual empresa é o melhor investimento pode ser obtida a partir dos lucros econômicos individuais?

Qual empresa é a melhor candidata - parte 2:

Calcular o lucro econômico das empresas combinadas (para AlbAm + Garciadores, fazer inicialmente apenas o primeiro cenário: em que a tecnologia 'sobrevivente' será a da Garciadores).

Obs.: Atenção com o cálculo do resultado bruto e do resultado operacional de (1) AlbAm + Carblue e (2) AlbAm + Garciadores. Estes não são obtidos por meio da soma

REAd | Porto Alegre - Edição 82 - № 3 - setembro/dezembro 2015 - p. 782-795 


\section{Ricardo Goulart Serra}

dos respectivos valores das empresas individuais. Também se deve ter atenção no cálculo do estoque da AlbAm + Garciadores, que não deve ser calculado como a soma dos estoques individuais, mas sim por meio do índice 'dias de estoque' da Garciadores (haverá uma melhor eficiência neste ativo).

Como os números indicam que houve perda de valor agregado com a combinação AlbAm + Garciadores, pode-se inferir que a nova tecnologia não é financeiramente mais vantajosa. Calcular, portanto, o cenário alternativo para a empresa combinada AlbAm + Garciadores, considerando que a tecnologia 'sobrevivente' será a da AlbAm.

Com base nestes novos cálculos, discutir: Qual é aparentemente a melhor candidata? A indicação de qual empresa é o melhor investimento pode ser obtida a partir dos lucros econômicos resultantes (das empresas combinadas)?

Obs.: Note que o lucro econômico da AlbAm + Carblue é R\$ 4,6MM, da AlbAm + Garciadores (considerando a tecnologia 'sobrevivente': a da Garciadores) é R\$ 2,8MM, o que levaria a crer que comprar a Carblue seria melhor. O lucro econômico da AlbAm + Garciadores (considerando a tecnologia 'sobrevivente': a da AlbAm) é R\$ 4,9MM, o que levaria a crer que comprar a Garciadores e manter a tecnologia da AlbAm seria melhor.

Atenção, pois ainda não há condições de responder qual a melhor candidata. Esta resposta depende de quanto será pago pelas empresas...

\section{Qual empresa é a melhor candidata - parte 3:}

Como identificar qual a melhor candidata e qual a melhor tecnologia a ser adotada?

Para responder, é necessário calcular o valor das empresas. A sugestão, no presente caso de ensino, é fazê-lo por lucro econômico (valor da empresa ou firm value é a soma do capital investido (neste caso, o ativo operacional total) com o valor presente da agregação de valor, ou seja, o valor presente dos lucros econômicos (Miller e Modigliani, 1961; O'Byrne, 1996; Tsuji, 2006 e Assaf Neto, 2014, p. 129)).

Para simplificação, considere que o preço a ser pago será o preço calculado individualmente para a Carblue e para a Garciadores (R\$ 64,1MM e R\$ 85,1MM, respectivamente).

Obs.: O texto do caso indica que os preços devem ser calculados considerando que os lucros econômicos serão constantes e para sempre.

Somando-se o valor da AlbAm - de R \$287,7MM - (a) ao valor individual da Carblue obtémse R\$ 351,8MM e (b) ao valor individual da Garciadores obtém-se R \$ 372,8MM.

REAd | Porto Alegre - Edição 82 - No 3 - setembro/dezembro 2015 - p. 782-795 


\section{CASO AlbAm S.A.}

Estes valores devem ser comparados aos valores das possíveis empresas combinadas: (a1) AlbAm + Carblue: R\$ 358,8 MM, (b1) AlbAm + Garciadores - tecnologia 'sobrevivente' Garciadores: R\$ 357,9 MM e (b2) AlbAm + Garciadores - tecnologia 'sobrevivente' AlbAm: R\$ 377,4 MM.

Para a Garciadores, deve-se promover um ajuste com relação ao estoque da empresa combinada, somando em (b1) $\mathrm{R} \$ 4,9 \mathrm{MM}$ caso a tecnologia sobrevivente seja a da Garciadores (pois haverá desinvestimento pela redução do estoque) ou subtraindo em (b2) $\mathrm{R}$ \$ 1,6 MM caso a tecnologia sobrevivente seja a da Garciadores (pois haverá um investimento pelo aumento do estoque).

Com estes cálculos, pode-se indicar a recomendação: comprar a Carblue, pois esta aquisição gerará $\mathrm{R} \$ 7,0 \mathrm{MM}$ (o total investido de $\mathrm{R} \$ 351,8 \mathrm{MM}$ comparado ao valor da empresa combinada de R \$358,8MM).

Obs.: Pode ser interessante insistir na discussão de a empresa melhor gerida não ser necessariamente o melhor investimento.

Outras possibilidades a serem exploradas - sugestão 1:

Como, conceitualmente, estudo de caso permite outras soluções, pode-se ter interesse em explorar outros cenários de empresas combinadas, a partir de índices financeiros a serem adotados para as empresas combinadas. 'Dias de Recebível' e Margem Operacional são 2 possibilidades.

Caso haja interesse, é possível calcular o valor das empresas (firm value) a partir do fluxo de caixa operacional (resultado operacional - imposto de renda operacional + depreciação CAPEX - investimento em capital de giro líquido).

Para que os valores calculados por lucro econômico e por fluxo de caixa operacional sejam iguais, é preciso manter as mesmas premissas. A premissa adotada, no cálculo do firm value por lucro econômico, é a de que o lucro econômico será constante, ou seja, o resultado operacional e os ativos operacionais totais serão constantes. Assim, no cálculo do firm value por fluxo de caixa operacional, deve-se considerar que o investimento líquido seja zero (depreciação - CAPEX - investimento em capital de giro líquido) e, portanto, o fluxo de caixa será o próprio resultado operacional líquido (resultado operacional - imposto operacional). Portanto, o firm value será o resultado operacional líquido dividido pelo WACC (uma perpetuidade sem crescimento).

REAd | Porto Alegre - Edição 82 - N 3 - setembro/dezembro 2015 - p. 782-795 


\section{Ricardo Goulart Serra}

Outras possibilidades a serem exploradas - sugestão 2:

Também é possível, ao final do caso, voltar à discussão de gestão por EBITDA ou gestão por valor. Verifique que a melhor solução não é a que promove o maior aumento de EBITDA.

Obs.: Compare os EBITDAs das empresas individuais, das empresas individuais somadas e das empresas combinadas.

\section{Outras possibilidades a serem exploradas - sugestão 3:}

Dependendo do nível e da profundidade do curso, o instrutor pode discutir artigos acadêmicos que investigaram o lucro econômico. Algumas sugestões seriam:

Artigos que evidenciam a superioridade do lucro econômico em relação a outras medidas: O'Byrne (1996) e Feltham et al (2004).

Artigos que não evidenciam a superioridade do lucro econômico em relação a outras medidas: Easton et al (1992), Biddle et al (1997), Kramer e Pushner (1997), Tsuji (2006), Kyriazis e Anastassis (2007) e Serra et al (2011).

O artigo de Chari (2009) indica diversos estudos sobre lucro econômico, classificando-os em 4 categorias: (1) relevância dos ajustes contábeis, (2) superioridade do lucro econômico, (3) medidas de performance baseadas no lucro econômico e (4) demais estudos.

O capítulo 13 do Copeland et al (2005) discute outras métricas de performance, tais como: expectation-based-management, fluxo de caixa e opções reais, que pode ser útil 


\section{CASO AlbAm S.A.}

Tabela 2 - Resultados consolidados. A+C significa AlbAm + Carblue; A+G (1) significa AlbAm + Garciadores (tecnologia Garciadores) e A+G (2) significa AlbAm + Garciadores (tecnologia AlbAm).

\begin{tabular}{|c|c|c|c|c|c|c|}
\hline [R\$ Milhões] & AlbAm & Carblue & Garciadores & $\mathbf{A}+\mathbf{C}$ & $A+G(1)$ & $A+G(2)$ \\
\hline \multicolumn{7}{|l|}{ Contas do Balanço } \\
\hline Caixa Operacional & 40,0 & 7,5 & 11,0 & 47,5 & 51,0 & 51,0 \\
\hline Contas a Receber & 65,0 & 16,0 & 20,0 & 81,0 & 85,0 & 85,0 \\
\hline Estoque & 45,0 & 11,5 & 12,5 & 56,5 & 52,6 & 59,1 \\
\hline Imobilizado & 170,0 & 43,0 & 53,0 & 213,0 & 223,0 & 223,0 \\
\hline Contas a Pagar & 35,0 & 9,0 & 10,7 & 44,0 & 45,7 & 45,7 \\
\hline Impostos a Pagar & 10,0 & 2,0 & 3,0 & 12,0 & 13,0 & 13,0 \\
\hline Salários e Enc. a Pagar & 5,0 & 2,0 & 2,3 & 7,0 & 7,3 & 7,3 \\
\hline [R\$ Milhões] & AlbAm & Carblue & Garciadores & $\mathbf{A}+\mathbf{C}$ & $A+G(1)$ & $A+G(2)$ \\
\hline \multicolumn{7}{|l|}{ DRE Parcial } \\
\hline Vendas Líquidas & 800,0 & 200,0 & 250,0 & $1.000,0$ & $1.050,0$ & $1.050,0$ \\
\hline (-) $\mathrm{CPV}$ & 600,0 & 154,0 & 185,0 & 750,0 & 777,0 & 787,5 \\
\hline (=) Resultado Bruto & 200,0 & 46,0 & 65,0 & 250,0 & 273,0 & 262,5 \\
\hline (-) Despesas Operacionais & 115,0 & 29,0 & 36,0 & 144,0 & 151,0 & 151,0 \\
\hline (=) Resultado Operacional & 85,0 & 17,0 & 29,0 & 106,0 & 122,0 & 111,5 \\
\hline Indicadores Selecionados & AlbAm & Carblue & Garciadores & $\mathbf{A}+\mathbf{C}$ & $A+G(1)$ & $A+G(2)$ \\
\hline Dias de Estoque & 27,4 & 27,3 & 24,7 & 27,5 & 24,7 & 27,4 \\
\hline Margem Bruta & $25,0 \%$ & $23,0 \%$ & $26,0 \%$ & $25,0 \%$ & $26,0 \%$ & $25,0 \%$ \\
\hline Lucro Econômico e Valor & AlbAm & Carblue & Garciadores & $\mathbf{A}+\mathbf{C}$ & $A+G(1)$ & $A+G(2)$ \\
\hline Resultado Operacional Líquido & 56,1 & 11,2 & 19,1 & 70,0 & 80,5 & 73,6 \\
\hline Capital de Giro Líquido & 100,0 & 22,0 & 27,5 & 122,0 & 122,6 & 129,1 \\
\hline Ativo Fixo & 170,0 & 43,0 & 53,0 & 213,0 & 223,0 & 223,0 \\
\hline Ativo Operacional Total & 270,0 & 65,0 & 80,5 & 335,0 & 345,6 & 352,1 \\
\hline ROIC & $20,8 \%$ & $17,3 \%$ & $23,8 \%$ & $20,9 \%$ & $23,3 \%$ & $20,9 \%$ \\
\hline Lucro Econômico & 3,4 & $(0,2)$ & 1,0 & 4,6 & 2,8 & 4,9 \\
\hline VP (Lucros Econômicos) & 17,7 & $(0,9)$ & 4,6 & 23,8 & 12,3 & 25,3 \\
\hline Valor da Empresa & 287,7 & 64,1 & 85,1 & 358,8 & 357,9 & 377,4 \\
\hline $\begin{array}{l}\text { Valor das Empresas Combinadas } \\
\text { Agregação de valor com a }\end{array}$ & & 351,8 & 372,8 & & & \\
\hline compra & & & & 7,0 & $(14,9)$ & 4,6 \\
\hline$(+/-) \Delta$ Estoque & & & & & 4,9 & $(1,6)$ \\
\hline Resultado Total & & & & 7,0 & $(10,0)$ & 3,0 \\
\hline
\end{tabular}

Fonte: Elaborada pelo autor. 


\section{Ricardo Goulart Serra}

\section{REFERÊNCIAS}

ASSAF NETO, A. Valuation: métricas de valor e avaliação de empresas. São Paulo: Atlas, 2014.

BERK, J.; DEMARZO, P. Finanças Empresariais. Porto Alegre: Bookman, 2008.

BIDDLE, G. C.; BOWEN, R. M.; WALLACE, J. S. Does EVA beat earnings? Evidence on associations with stock returns and firm value. Journal of Accounting and Economics, v. 24, n. 3, pp. 301-336, 1997.

CHARI, L. Measuring value enhancement through economic value added: evidence from literature. IUP Journal of Applied Finance, v. 15, n. 9, pp. 46-62, 2009.

COPELAND, T.; WESTON, J. F.; SHASTRI, K. Financial Theory and Corporate Policy. $4^{\mathrm{a}}$ ed. Boston: Pearson, 2005.

EASTON, P. D.; HARRIS, T. S.; OHLSON, J. A. Aggregate accounting earnings can explain most of security returns: the case of long run intervals. Journal of Accounting and Economics, v. 15, n. 2, pp. 119-142, 1992.

EHRHARDT, M.C.; BRIGHAM, E.F. Administração Financeira: Teoria e Prática. $2^{\mathrm{a}}$ ed. São Paulo: Cengage Learning, 2012.

FELTHAM, G. D.; ISAAC, G. E.; MBAGWU, C.; VAIDYANATHAN, G. Perhaps EVA does beat earnings: revisiting previous evidence. Journal of Applied Corporate Finance, v. 16, n. 1, pp. 83-88, 2004.

KRAMER, J. K.; PUSHNER, G. An empirical analysis of economic value added as a proxy for market value added. Financial Practice and Education, v. 7, n. 1, pp. 41-49, 1997.

KYRIAZIS, D.; ANASTASSIS, C. The validity of the economic value added approach: an empirical application. European Financial Management, v. 13, n. 1, pp. 71-100, 2007.

MILLER, M.; MODIGLIANI, F. Dividend policy, growth, and the valuation of shares. Journal of Business, v. 34, n. 4, pp. 411-433, 1961.

O'BYRNE, S.F. EVA and market value. Journal of Applied Corporate Finance, v. 9, n. 1, pp. 116-125, 1996.

SERRA, R. G.; MARTELANC, R.; SECURATO, J. R. Does economic profit beat earnings? Evidence from Brazil. IUP Journal of Applied Finance, v. 17, n. 4, pp. 68-81, 2011.

TSUJI, C. Does EVA beat earnings and cash flow in Japan? Applied Financial Economics, v. 16, pp. 1199-1216, 2006.

REAd | Porto Alegre - Edição 82 - N 3 - setembro/dezembro 2015 - p. 782-795 